\title{
Les interfaces Cerveau-Machine pour la palliation du handicap moteur sévère
}

\author{
Marie-Hélène Bekaert* - Claudine Botte-Lecocq* - François \\ Cabestaing* - Alain Rakotomamonjy** \\ * LAGIS - UMR CNRS 8146. Université des Sciences et Technologies de Lille, \\ Cité scientifique, 59655 Villeneuve d'Ascq Cedex \\ francois.cabestaing@univ-lille1.fr \\ ** LITIS - EA 4051. INSA de Rouen, \\ Avenue de l'Université - BP 8. 76801 Saint-Étienne-du-Rouvray Cedex
}

\begin{abstract}
RÉSUMÉ. Les interfaces cerveau-machine (BMI: Brain-Machine Interface) sont des systèmes de communication directe entre un individu et une machine ne reposant pas sur les canaux de communication standard que sont nos nerfs périphériques et nos muscles. Dans une BMI, l'activité cérébrale de l'utilisateur est enregistrée, analysée et traduite en commandes destinées à la machine. Nous présentons quelques caractéristiques de l'activité cérébrale qui peuvent être exploitées comme source d'information dans une BMI. Ensuite, nous décrivons les principales approches de traitement et de classification des signaux mises en æuvre dans les BMIs. Nous présentons enfin un état de l'art des différentes interfaces BMI développées jusqu'alors, en nous attachant plus particulièrement à celles dédiées à l'aide aux personnes atteintes d'un handicap moteur sévère dans leur tâche de communication ou de contrôle de machines.

ABSTRACT. Brain-Machines Interfaces are direct communication devices between a person and a machine that do not rely on the activation of peripheral nerves or muscles. Brain-Machine Interfaces are based on the acquisition and analysis of the brain activity which is then converted to control signals for the device. After presenting the brain activity characteristics that can be exploited as information sources, we describe the most common approaches to signal acquisition and signal classification used in a BMI. Then, we present a state of the art of the present BMI devices specifically designed for helping severely handicapped people to communicate and control systems.
\end{abstract}

MOTS-CLÉS : Interface machine ordinateur, BMI, interface cerveau-machine, BCI, aide au handicap, communication palliative

KEYWORDS: Brain-Machine Interface, BMI, BCI, Assistive technology, Palliative Communication

$2^{\mathrm{e}}$ soumission à $S T H$, le 20 novembre 2009 


\section{Introduction}

Certains patients victimes d'un accident vasculaire cérébral grave restent dans un état de paralysie musculaire complète (LIS : Locked-In Syndrom). Le même handicap moteur très sévère touche les personnes atteintes de sclérose latérale amyotrophique (SLA ou maladie de Lou Gehrig) durant les derniers stades de la maladie. Dans la plupart des cas, ces affections laissent toutes leurs facultés mentales aux personnes qui en sont atteintes, mais leur interdit toute forme de communication avec leur entourage. Les interfaces cerveau-machine sont l'approche qui semble actuellement la plus prometteuse pour pallier ce handicap.

Une interface cerveau-machine, ou BMI (Brain-Machine Interface), est constituée des éléments suivants, schématisés sur la figure 1 : en entrée, on trouve un soussystème chargé d'acquérir et de numériser les signaux liés à l'activité cérébrale de l'utilisateur; ensuite, un sous-système est chargé de traiter ces signaux et de les traduire en commandes envoyées à la machine; enfin, une partie des résultats est présentée à l'utilisateur sous une forme aisément compréhensible afin de constituer une boucle permettant l'apprentissage (bio-feedback).

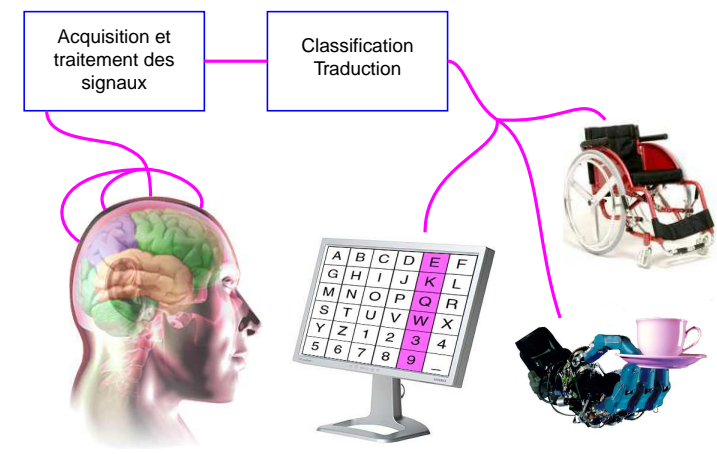

Figure 1. Structure d'une interface cerveau-machine

L'activité cérébrale d'un individu se manifeste par l'apparition de potentiels électriques et de champs magnétiques au niveau de sa tête. Bien que certains chercheurs aient envisagé de détecter les champs magnétiques créés par l'activité du cerveau (Georgopoulos et al., 2005; Mellinger et al., 2007), ou d'utiliser des techniques récentes d'imagerie fonctionnelle (Sitaram et al., 2008), ce sont souvent les potentiels électriques qui sont enregistrés dans le cas des BMIs. Cette mesure est réalisée soit de manière non invasive au niveau du cuir chevelu (scalp-EEG : électroencéphalogramme), soit à la surface du cortex (ECoG : électrocorticogramme) ou par l'intermédiaire d'électrodes implantées directement dans le cerveau. Ces deux dernières techniques d'acquisition de l'activité cérébrale sont qualifiées d'invasives du fait qu'elles nécessitent une opération chirurgicale afin d'introduire un système de mesure à l'intérieur du crâne du patient. 
Les interfaces BMI qualifiées d'asynchrones analysent les amplitudes, fréquences ou phases des rythmes cérébraux normalement reliés à l'activité volontaire de l'individu, lesquels sont notamment détectés dans l'EEG à l'aplomb des aires sensorimotrices du cortex (Wolpaw et al., 2004). Par exemple, l'amplitude du rythme $\mu$ se modifie non seulement durant un mouvement réel, mais également quand l'individu imagine qu'il réalise un mouvement. Une autre approche, initialement proposée par Farwell et Donchin (Farwell et al., 1988), est utilisée dans certaines BMI qualifiées de synchrones. Elle consiste à détecter dans les signaux EEG des potentiels évoqués par des stimuli perçus par l'individu (ERP : Event Related Potentials). L'interface génère les stimuli puis détecte dans les signaux les potentiels évoqués par ces derniers. La description de ces deux approches fait l'objet de la deuxième partie de cet article.

Les signaux mesurant l'activité électrique cérébrale ont des caractéristiques très diverses selon le niveau d'invasivité de l'interface. Les techniques invasives mesurent les potentiels d'action, signaux très brefs (spikes) et relativement simples car similaires à des impulsions, enregistrés sur l'axone d'un neurone individuel. De telles interfaces fournissent d'excellents résultats tant au niveau de la mesure qu'au niveau du décodage de l'activité cérébrale. Néanmoins, ces systèmes sont actuellement peu utilisés dans le cadre de la palliation du handicap car encore controversés. En effet, ils causent sur les tissus des dommages irréversibles, et peuvent engendrer des risques d'infection. De plus, la durée de vie des électrodes implantées est limitée. De ce fait, la plupart des expérimentations d'interfaces BMI invasives ont été réalisées jusqu'alors sur des animaux, et particulièrement sur des primates dont la neuroanatomie du cortex moteur est assez proche de celle de l'homme (Souères et al., 2007). A l'opposé, au sein des interfaces non invasives, les signaux enregistrés à la surface du crâne résultent de la superposition des activités électriques de plusieurs millions de neurones ce qui rend difficile la localisation de l'activité cérébrale. De plus, comme l'analyse directe de ces signaux de faible intensité est très complexe, des techniques très variées visant à améliorer la qualité des signaux sont proposées dans la littérature (Bashashati et al., 2007). Elles sont décrites dans la troisième partie de cet article.

Il convient ensuite d'extraire des signaux prétraités un nombre limité d'éléments caractéristiques (ou attributs) concentrant la majeure partie de l'information utile initiale. Une méthode de classification de données exploitant ces éléments caractéristiques peut alors permettre d'identifier l'état d'activité cérébrale de l'individu. Les techniques d'extraction d'attributs et de classification utilisées dans les BMIs sont très variables selon que l'interface est de type synchrone ou asynchrone (Lotte et al., 2007). Ces méthodes sont présentées dans la quatrième partie.

A l'issue de l'étape précédente, les informations extraites sont traduites en signaux de commande, permettant ainsi une communication élaborée entre l'individu et la machine. Quelles que soient les techniques d'acquisition et de traitement de signaux développées, l'utilisation d'une BMI par un patient nécessite plusieurs phases. Durant la phase de préparation, le patient est installé confortablement et les électrodes permettant d'acquérir les signaux cérébraux sont reliées au matériel d'acquisition qui est 
alors calibré. Ensuite, deux types de phases d'apprentissage rentrent en jeu : une phase d'adaptation de l'interface BMI au patient et une phase d'entraînement du patient.

La phase d'adaptation de l'interface BMI au patient dépend du type d'interface utilisé et du principe de classification des signaux. Dans le cas d'une interface synchrone, cette phase d'adaptation consiste à générer des signaux EEG liés aux états mentaux et à entraîner la machine à reconnaître ces états. Cette phase nécessite généralement une vingtaine de minutes. Ce principe d'adaptation de l'interface BMI au patient sans nécessité d'entraînement du patient a également été validé par Blankertz et al. dans le cadre d'interfaces BMI asynchrones (Blankertz et al., 2006a).

Durant la phase d'entraînement, le patient apprend à contrôler l'interface BMI. Cette phase, simple à mettre en oeuvre et particulièrement adaptée aux interfaces asynchrones, est basée sur la capacité de l'utilisateur à apprendre à changer volontairement certaines caractéristiques de son signal EEG. Cet apprentissage peut nécessiter plusieurs heures. Généralement, l'interface BMI propose au patient un bio-feedback ayant une dépendance aux caractéristiques de l'EEG (Wolpaw et al., 2000), (McFarland et al., 2000), (Birbaumer et al., 2000). Ce retour permet au patient de s'adapter au système de reconnaissance de la machine et ainsi de pouvoir améliorer ses performances de contrôle de l'interface BMI.

Actuellement, ces deux approches peuvent être considérées en opposition : la première focalise l'essentiel du contrôle de l'interface BMI sur la capacité de la machine à reconnaître des états mentaux du patient. Dans le deuxième cas, c'est le sujet qui porte toute la charge du contrôle de l'interface BMI : c'est sa capacité à se concentrer et à contrôler son EEG qui permet un bon fonctionnement de l'interface BMI. Une perspective essentielle pour les BMI serait donc la mise en oeuvre conjointe, en temps réél et au cours de l'utilisation, de ces deux types d'apprentissage. Le but serait alors que le patient continue à améliorer sa façon d'appréhender l'interface BMI et que la machine s'adapte au patient au fur et à mesure de son utilisation.

Dans la cinquième partie de cet article, nous décrivons les principales applications actuelles des BMI dans le domaine des technologies de l'assistance que sont l'aide à la communication pour les patients atteints de paralysie musculaire complète, la récupération du mouvement pour les personnes présentant une infirmité motrice par le contrôle par la pensée d'un fauteuil roulant, d'une orthèse ou d'une prothèse, et plus récemment l'aide au diagnostic et à la récupération fonctionnelle. Plusieurs auteurs ont proposé un état de l'art des techniques et applications BMIs (Wolpaw et al., 2002), (Pfurtscheller et al., 2006a), (Lebedev et al., 2006), (Schwartz et al., 2006), les plus récents proposant plus de 200 références traitant des BMIs (Mason et al., 2007).

Enfin, nous concluons sur l'état d'avancement des recherches et présentons quelques perspectives à court et moyen terme de la recherche sur les BMIs. 


\section{BMI asynchrones et synchrones}

Lors de l'utilisation d'une BMI asynchrone, l'individu interagit avec le système quand il le décide, en modifiant de façon volontaire son activité cérébrale. La BMI détecte cette modification dans les signaux EEG et la transforme en commandes. Habituellement, dans les BMI asynchrones, les signaux de commande sont continus, ce qui signifie qu'ils permettent un contrôle progressif des éléments présents dans l'interface, par exemple la position d'un curseur. Plusieurs signaux découlant de l'activité cérébrale sont utilisés dans les interfaces asynchrones :

Potentiels corticaux lents (SCPS : Slow Cortical Potential Shifts). Les SCPSs sont des variations très progressives du potentiel cortical moyen, durant de quelques centaines de millisecondes jusqu'à plusieurs secondes. La plupart des individus peuvent apprendre à contrôler leur potentiel cortical afin de provoquer une variation positive ou négative, qui est ensuite transformée en commande par la BMI. Un retour d'information visuel (bio-feedback), généralement un affichage sous forme simple de son potentiel cortical, est fourni à l'utilisateur afin de l'aider durant le processus d'apprentissage (Birbaumer et al., 2000).

Activité oscillatoire sensorimotrice. L'activité cérébrale enregistrée à l'aplomb du cortex sensorimoteur se modifie durant l'activité motrice, lors de la planification du mouvement ou encore pendant que l'individu imagine qu'il réalise un mouvement. Plus précisément, l'énergie des signaux dans la bande de fréquences $\mu(8-15 \mathrm{~Hz})$ et la bande $\beta(15-35 \mathrm{~Hz})$ décroît dans l'hémisphère opposé au côté où se déroule le mouvement (controlatéral) et augmente dans l'autre (ipsilatéral). Les changements, en amplitude et en fréquence, sont très variables d'un individu à l'autre, et ils évoluent fortement au cours du temps. Grâce au bio-feedback, il est également possible d'apprendre à contrôler ces variations dans le cas de mouvements imaginés, afin de piloter une BMI à un ou deux degrés de liberté (Wolpaw et al., 2004).

Signaux EEG spontanés. Quand un individu réalise une tâche mentale, autre que celle correspondant à des mouvements réels ou imaginés, son activité électrique cérébrale se trouve également modifiée. Des tâches complexes, qui entraînent l'activation de larges groupes de neurones, comme le calcul mental ou le fait d'imaginer des mouvements d'objets dans l'espace, ont une influence non négligeable sur les signaux EEG (Jerbi et al., 2007). L'activation de zones spécifiques du cerveau peut être identifiée par la BMI durant une phase d'apprentissage afin d'être ensuite utilisée pour commander la machine (del R. Millán et al., 2002; Le Pavec et al., 2008).

Dans une BMI synchrone, ce n'est pas l'activité spontanée du cerveau qui est enregistrée, mais sa réponse à un stimulus. Cette dernière est détectée dans les signaux puis transformée en commande. Du fait que cette réponse cérébrale est une caractéristique innée de l'individu, l'utilisation de ce type de BMI nécessite en général un apprentissage très limité. Deux types principaux de réponses cérébrales sont utilisés dans les BMIs synchrones : 
Potentiels évoqués visuels de bas-niveau (SSVERs : Steady State Visual Evoked Responses). Les SSVERs apparaissent normalement dans le cortex visuel primaire après des stimuli visuels de fréquence préfixée. Des formes très texturées, comme des damiers comportant des cases blanches et noires, dont le contraste est modulé à une fréquence fixe, entraînent l'apparition de très forts potentiels de type SSVER. Ces derniers se manifestent comme une augmentation de l'amplitude du signal EEG dans la bande de fréquence correspondant à celle du stimulus. Les utilisateurs peuvent apprendre à contrôler l'amplitude de leurs SSVERs grâce au bio-feedback et ensuite utiliser ce talent pour interagir avec la BMI (del R. Millán et al., 2002), (Gao et al., 2003), (Lüth et al., 2007), (Friman et al., 2007), (Müller-Putz et al., 2008).

Potentiels évoqués par un événement (ERPs : Event Related Potentials). Les ERPs sont des signaux électriques de faible durée qui sont engendrés par une réponse du cerveau à des stimuli extérieurs, qu'ils soient visuels, auditifs ou encore tactiles. Le temps de latence séparant le stimulus de l'ERP dépend de la complexité de la tâche mentale que le stimulus a suscité. Par exemple, les ERPs de type P300 apparaissent environ 300 millisecondes après le stimulus, d'où leur appellation. Ils sont reliés à une tâche cognitive comme le comptage des apparitions d'un type particulier de stimulus. Dans les BMIs exploitant les ERPs, des motifs spatio-temporels spécifiques à l'individu et décrivant son activité cérébrale après stimulus sont détectés dans les signaux EEG puis utilisés afin de générer des commandes binaires (Farwell et al., 1988), (Bayliss, 2003), (Wang et al., 2005).

Dans les BMIs synchrones, les stimuli sont présentés à l'utilisateur à une cadence très rapide afin d'augmenter le débit d'informations permettant la communication. Le système détecte les réponses aux stimuli en classant des éléments caractéristiques extraits des signaux EEG. Ces interfaces délivrent des signaux tout ou rien, ce qui revient à actionner un interrupteur, qui permettent de sélectionner une option parmi plusieurs pour le fonctionnement de la machine.

\section{Prétraitement des signaux}

L'étape de prétraitement a deux principaux objectifs, à savoir l'élimination des artéfacts et l'amélioration du rapport signal sur bruit des signaux. Dans le cas des signaux EEG enregistrés sur le scalp, les artéfacts proviennent de potentiels électriques parasites engendrés par des activités de l'individu autres que celle visant à piloter l'interface : mouvements des muscles — électromyogramme : EMG, électrocardiogramme : ECG, ou encore électro-oculogramme : EOG — clignements des paupières, etc... Les signaux sont également entachés d'un bruit résultant de la superposition des champs électriques issus de larges populations de neurones dont l'activité n'est pas associée à la tâche réalisée par l'individu.

Parmi les techniques de prétraitement, certaines font intervenir une combinaison des signaux issus de plusieurs électrodes, ce qui s'apparente à un filtrage spatial. Elles exploitent le fait que les sources d'informations utiles sont en général assez 
localisées alors que les sources de bruit sont plus diffuses. De ce fait, un filtre spatial de type passe-haut conserve les informations et atténue le bruit. Parmi les filtres simples, le plus utilisé est certainement le Laplacien introduit initialement par McFarland (McFarland et al., 1997), qui consiste à soustraire à un signal la moyenne des signaux recueillis sur les électrodes voisines. Cette méthode est beaucoup plus efficace que le fait de soustraire la moyenne calculée sur la totalité des électrodes (Common Average : CAR).

Des techniques de filtrage spatial plus élaborées, mais qui nécessitent une adaptation à l'individu et même parfois aux conditions d'acquisition des signaux, ont été décrites dans la littérature (Bashashati et al., 2007). On peut citer l'Analyse en Composantes Principales (ACP) ou en Composantes Indépendantes (ACI) dont l'objectif est de séparer les sources de bruit des sources d'information par un filtrage dont les coefficients sont calculés à partir de signaux d'une base d'apprentissage. La méthode CSP (Common Spatial Pattern), qui permet de calculer les coefficients du filtre spatial en recherchant des structures organisées dans l'ensemble des signaux, a également été utilisée récemment (Wei et al., 2005). Il faut souligner que ces méthodes restent partiellement supervisées, du fait que les signaux significatifs doivent être sélectionnés parmi tous les résultats disponibles.

Les techniques de reconstruction et de localisation de sources peuvent également être considérées comme des prétraitements (Congedo et al., 2006), (Besserve et al., 2008). Leur objectif est de calculer une estimation de l'activité corticale de l'individu à partir des mesures EEG. Cela consiste à résoudre un problème inverse, à savoir la détermination des positions et orientations des dipôles électriques élémentaires à partir du champ électrique résultant de la combinaison de leurs effets. Plusieurs techniques relevant de cette approche ont été appliquées avec succès au prétraitement des signaux dans les BMIs, notamment l'algorithme sLORETA (Standardized low resolution brain electromagnetic tomography) (Pascual-Marqui, 2002).

\section{Classification des signaux}

Deux présentations de l'état de l'art concernant la classification des signaux dans les BMIs ont été publiées récemment (Bashashati et al., 2007; Lotte et al., 2007). Du fait du haut degré de complexité de ce problème - haute dimensionalité, apprentissage parfois non supervisé et/ou taille limitée de l'ensemble d'apprentissage — de nombreux travaux ont été menés par les équipes spécialisées dans la classification automatique de données. Les deux étapes clés sont l'extraction de vecteurs caractéristiques discriminants à partir des données brutes, et la classification réalisée sur la base de ces vecteurs. Les méthodes décrites dans la littérature concernant ces deux étapes sont très différentes selon qu'il s'agit de classer les données issues d'une BMI asynchrone ou synchrone.

Dans le cas des interfaces asynchrones, la plupart des méthodes d'extraction de vecteurs caractéristiques exploitent les connaissances acquises par les neurophysiolo- 
gistes. Le traitement vise alors à mettre en évidence une propriété des signaux liée à une activité cérébrale particulière. Par exemple, dans les BMIs qui exploitent les variations temporelles des potentiels corticaux lents, un simple filtrage passe-bas est utilisé, alors que dans les interfaces utilisant les rythmes sensorimoteurs, il s'agira plutôt d'extraire la puissance du signal dans la gamme de fréquences du rythme considéré. Ces caractéristiques sont mises en évidence au moyen de transformations temps-fréquence ou encore par des filtres auto-régressifs (Bashashati et al., 2007).

La classification des signaux est ensuite réalisée sur la base de vecteurs caractéristiques définis dans un espace de relativement faible dimension. Dans certains cas très simples, l'étape de classification consiste même en un simple seuillage d'un signal mis en évidence lors du prétraitement (McFarland et al., 1997). En règle générale, dans le cas des BMIs asynchrones, l'objectif de l'étape de classification est de déterminer l'état mental de l'individu. Cele ne pose pas de problème particulier lorsque les états mentaux potentiels sont connus par avance, c'est à dire quand la technique de classification peut être supervisée (Lotte et al., 2007).

Dans le cas des interfaces synchrones, comme les réponses à un stimulus varient très fortement d'un individu à l'autre, les caractéristiques discriminantes sont souvent extraites des signaux par apprentissage. Les données de l'ensemble d'apprentissage sont analysées soit dans le domaine temporel, soit dans un domaine transformé, par exemple après une transformation en ondelettes. A ce niveau de l'analyse, le principal problème rencontré est celui de la réduction des dimensions de l'espace contenant les vecteurs caractéristiques (Krusienski et al., 2006).

Par la suite, la classification fait habituellement intervenir seulement deux classes : celle caractérisant l'état standard de l'individu et celle décrivant les réponses effectives à un stimulus qualifiées de cibles. Comme le rapport signal sur bruit des signaux initiaux est très faible, les deux classes se recouvrent fortement, et même des classifieurs très performants aboutissent à un taux d'erreur non négligeable. De ce fait, les traitements sont réitérés sur un nombre plus ou moins important de réponses successives afin de diminuer l'influence du bruit par moyennage.

Quand la dimension de l'espace des caractéristiques est élevée, comme lorsque le vecteur caractéristique est constitué de tous les échantillons de toutes les électrodes dans le domaine temporel, un simple classifieur linéaire permet théoriquement de bien séparer les classes. En revanche, l'apprentissage des paramètres de ce classifieur est problématique du fait que le nombre d'éléments de l'ensemble d'apprentissage est faible en regard de la dimension de l'espace (Krusienski et al., 2006). A l'opposé, quand on limite au préalable la dimension du vecteur caractéristique par des transformations adaptées, les classifieurs non-linéaires — notamment les méthodes à noyaux - ou les combinaisons de classifieurs sont bien plus performants (Rakotomamonjy et al., 2005). 


\section{Prototypes opérationnels de palliation du handicap moteur sévère}

\subsection{Communication palliative}

Les interfaces BMI de communication palliative ne sont autres que des claviers virtuels permettant à un individu de saisir un texte sans la moindre activité musculaire. La difficulté essentielle d'un tel système de communication palliative est de faire correspondre un petit nombre d'états mentaux - extraits de l'analyse des signaux cérébraux — à un grand nombre de symboles d'écriture (26 lettres, 10 chiffres, ainsi que des signes de ponctuation), compte tenu du faible rapport signal/bruit du signal d'entrée. Dans cette section, nous présentons les BMI de communication palliative les plus répandues, qui diffèrent avant tout par le paradigme de communication homme/machine qu'elles exploitent ainsi que par leur niveau d'expérimentation.

Le P300 speller ou « matrix speller », initialement proposé par Farwell et Donchin (Farwell et al., 1988), est une interface de communication BMI non-invasive synchrone qui permet à l'utilisateur de saisir un texte en remplaçant le traditionnel clavier AZERTY par une matrice $6 \times 6$ de 36 symboles (voir figure 2). Les lignes et les colonnes de cette matrice sont intensifiées dans un ordre aléatoire, plusieurs fois par seconde. L'utilisateur, qui focalise son attention sur un symbole particulier, doit compter le nombre d'intensifications qui affectent ce symbole. Cette tâche cognitive de comptage entraîne la génération d'ERPs de type P300 automatiquement détectés par l'interface BMI, ce qui permet à cette dernière d'identifier la ligne et la colonne contenant le symbole sélectionné.

Un tel système permet à une personne valide d'épeler deux à trois lettres par minute. Depuis 1988, de nombreuses améliorations ont été apportées au P300 Speller, aussi bien en termes de techniques d'acquisition et de classification qu'en termes d'adaptation aux utilisateurs dans la phase de calibration de l'interface BMI (Donchin et al., 2000), (Li et al., 2006), (Liang et al., 2008). Les résultats obtenus lors de l'utilisation du P300 Speller par des patients atteints du syndrome d'enfermement sont relatés dans (Sellers et al., 2006b). Enfin, les effets de la taille de la matrice et de l'intervalle de temps séparant deux séquences successives sur les performances du P300 Speller en mode ligne ont été étudiés dans (Sellers et al., 2006a).

L'interface TTD (Thought Translation Device) est de type non invasive asynchrone détectant les variations des potentiels corticaux lents (Birbaumer et al., 2000). Le principe général de cette interface, mise au point à l'intention de sujets atteints de SLA, est de saisir un caractère par dichotomies successives de l'alphabet (voir figure 3). A chaque niveau de dichotomie, l'utilisateur sélectionne la partie de l'alphabet qui contient la lettre qu'il cherche à saisir en générant une variation de son potentiel cortical lent. L'interface BMI reconnaît alors cette variation, ce qui entraîne une division de la partie de l'alphabet ainsi sélectionnée en deux nouvelles sous-parties. Le processus de sélection est réitéré jusqu'à ce que l'utilisateur soit amené à ne sélectionner qu'un caractère parmi deux choix, entraînant l'affichage du caractère retenu en feedback. 


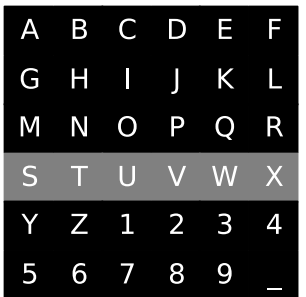

(a) Intensification d'une ligne

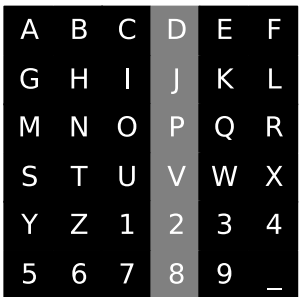

(b) Intensification d'une colonne

Figure 2. Matrice du P300 Speller.

Dans (Birbaumer et al., 2000), les auteurs relatent que l'utilisation de cette interface par des patients atteints de SLA leur a permis de communiquer avec le monde extérieur à la vitesse moyenne d'une lettre toutes les 2 minutes durant plusieurs mois. Depuis son développement, le TTD a été agrémenté d'un LSP (LSP : Language Support Program) qui permet une large sélection de lettres dans plusieurs langues (Hinterberger et al., 2003). De plus, Pham et al. proposent de remplacer les stimuli visuels ainsi que les informations visuelles de feedback par des stimuli et feedbacks auditifs (Pham et al., 2005).

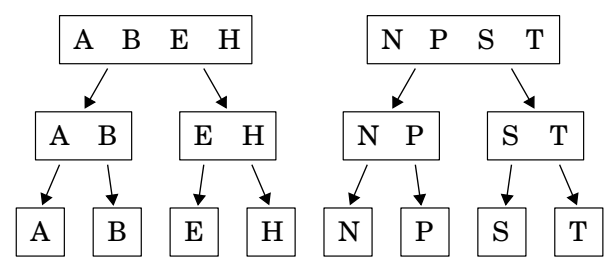

Figure 3. Sélection dichotomique du TTD (image extraite de (Kübler et al., 1999)).

Claviers virtuels sur la base de l'interface Graz-BCI. Dans (Obermaier et al., 2003), les auteurs utilisent ce même principe de dichotomies successives de l'alphabet pour proposer une interface de communication palliative non-invasive asynchrone basée sur l'analyse de l'activité sensorimotrice en utilisant l'interface standard GrazBCI (Guger et al., 2001; Pfurtscheller et al., 2000b). Sept niveaux de dichotomie sont nécessaires pour sélectionner puis valider la sélection d'une lettre parmi les 26 de l'alphabet. Cette technique nécessite une phase d'apprentissage au cours de laquelle il est demandé à l'utilisateur d'imaginer à plusieurs reprises un mouvement soit de la main gauche soit de la main droite, afin d'adapter les paramètres de l'interface BMI à l'utilisateur.

Jusqu'alors, seules des personnes valides ont testé ce système, et ont pu saisir correctement leur texte à la vitesse moyenne de 1,02 caractères par minute. Scherer $e t$ $a l$. proposent une interface basée sur le même principe de détection des signaux EEG 
dans laquelle l'utilisateur est amené à imaginer trois mouvements (main droite, main gauche, et pied), entraînant la nécessité d'adapter l'interface Graz-BCI à la détection de trois classes (Scherer et al., 2004). Dans cette interface, tant que l'utilisateur imagine un mouvement de pied, les lettres de l'alphabet défilent dynamiquement sur 2 colonnes du bas vers le haut de l'écran. Une fois le défilement arrêté, l'utilisateur choisit de sélectionner le caractère situé soit en haut à gauche de l'écran soit en haut à droite selon le côté du mouvement de main qu'il imagine. Cette amélioration a permis de faire passer la vitesse de communication à une moyenne de trois caractères corrects par minute en moyenne pour des personnes valides.

L'interface de communication palliative Hex-o-Spell, basée sur l'analyse des variations spontanées des signaux EEG issue de l'interface standard Berlin Brain Computer Interface (Blankertz et al., 2006a), est de type non invasive asynchrone. Pour piloter cette interface de communication présentée dans (Blankertz et al., 2006b; Blankertz et al., 2007), l'utilisateur doit imaginer deux mouvements que sont le mouvement de la main droite et le mouvement du pied droit. L'interface utilisateur, illustrée sur la figure 4, est composée de 6 hexagones contenant chacun 5 symboles (lettres ou signes de ponctuation), entourant un cercle dans lequel se trouve une flèche dont le déplacement circulaire permet la saisie du texte. Lorsque l'utilisateur imagine le mouvement de la main droite, la flèche (de longueur faible) tourne dans le sens des aiguilles d'une montre (voir figure 4(a)), tandis que lorsqu'il imagine le mouvement du pied droit, la longueur de la flèche augmente jusqu'à toucher et donc sélectionner l'hexagone correspondant (voir figure 4(b)). Après sélection, tous les autres hexagones disparaissent de l'écran pour laisser place à 6 hexagones contenant, pour 5 d'entre eux, l'une des 5 lettres contenues dans l'hexagone précédemment sélectionné (voir figure 4(c)). La procédure de sélection d'un hexagone précédemment décrite doit donc être itérée une seconde fois afin de sélectionner la lettre désirée. La vitesse de saisie de volontaires valides ayant testé cette interface de communication varie de 2,3 à 7 caractères par minute.

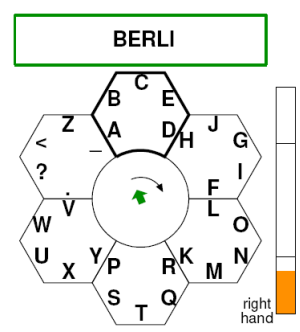

(a) phase de rotation

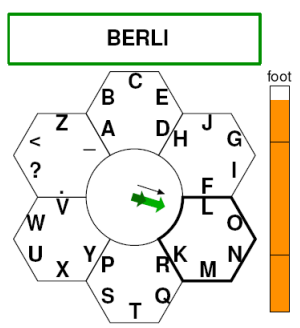

(b) phase de sélection

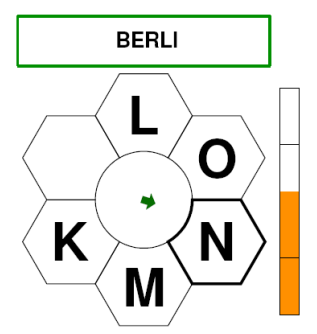

(c) nouvelles options

Figure 4. Ecran de saisie de l'interface Hex-o-Spell dans les différentes phases de la sélection d'un caractère (images extraites de (Blankertz et al., 2007)).

Le DASHER-BCI. Récemment, S. Wills a suggéré d'adapter l'interface de communication DASHER initialement proposée par D. Ward et D. MacKay (Ward et 
al., 2000) aux besoins des personnes présentant un handicap moteur sévère, en y insérant une composante BMI (Wills et al., 2006). Le DASHER utilise un modèle de langage qui prédit la probabilité d'apparition de chacune des lettres en fonction du contexte, et lui alloue un espace à l'écran en conséquence, comme illustré sur les figures 5(a) et 5(b). Le DASHER-BCI proposé par Felton et al. est une interface de communication palliative de type non-invasive asynchrone basée sur l'activité oscillatoire sensorimotrice (Felton et al., 2007). La phase d'apprentissage consiste tout d'abord à demander à l'utilisateur d'imaginer différents mouvements et de sélectionner les deux mouvements imaginés les plus discriminants. L'utilisateur est alors entraîné à contrôler le déplacement rectiligne d'un curseur à l'écran à partir de l'imagination de ces deux mouvements lui permettant ainsi de contrôler la navigation dans le paysage du DASHER. Le système utilisé pour l'interfaçage BMI est le BCI'2000 (Schalk et al., 2004).

Les auteurs rapportent les résultats de l'expérimentation de ce DASHER-BCI par 5 personnes valides. Les performances obtenues montrent que le DASHER-BCI est une interface de communication potentielle pour les personnes victimes d'un handicap moteur sévère. La difficulté majeure se situe au niveau de l'attention et du contrôle permanents que le pilotage du système nécessite. Il est aussi important de noter que les auteurs concluent que le DASHER-BCI n'est pas encore prêt à être directement utilisable par des personnes handicapées.

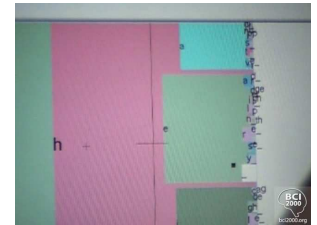

(a) Le Paysage DASHER

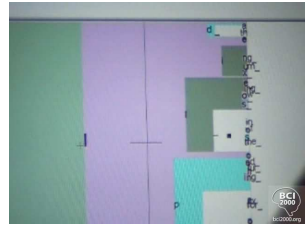

(b) Déplacement du curseur

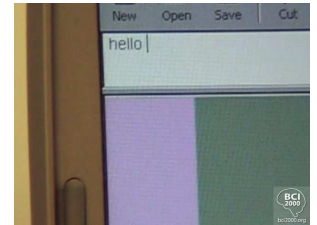

(c) Mot "hello" épelé en - de 30s

Figure 5. Contrôle de la position verticale du curseur dans le paysage du système DASHER par une BCI (images extraites d'une vidéo du site web BCI2000).

\subsection{Pilotage d'un fauteuil roulant par une interface BMI}

Tandis que certaines interfaces BMI visent à améliorer la communication des personnes atteintes d'un handicap moteur sévère avec le monde extérieur, d'autres sont développées pour augmenter leur autonomie. C'est ainsi que plusieurs équipes se concentrent actuellement sur le développement de fauteuils roulants pilotés par l'activité cérébrale via une BMI.

Tanaka et al., Université de Tokyo ont été les premiers à proposer un fauteuil roulant électrique dont la direction du déplacement est commandée par la pensée (Tanaka et al., 2005b). L'interface BMI utilisée est de type non invasive asynchrone basée sur l'analyse des signaux EEG spontanés dans la bande 0,5 - $30 \mathrm{~Hz}$. Bien que les auteurs 
n'explicitent pas le type d'imagerie mentale demandée aux utilisateurs, ils constituent un ensemble d'apprentissage de taille limitée permettant à l'interface BMI de discriminer les 2 déplacements imaginés droite et gauche (Tanaka et al., 2005a).

Le fauteuil, ainsi équipé de l'interface BMI, a été testé par 6 personnes valides dont la tâche était d'atteindre l'une ou l'autre des 2 zones dites cibles d'une pièce vide (virtuellement divisée en 28 rectangles de $60 \times 90 \mathrm{~cm}$ ), ces 2 zones étant respectivement situées à l'extrême gauche et à l'extrême droite de la pièce (voir figure 6). A chaque imagination de mouvement détecté s'en suit un déplacement de 45 degrés dans la direction reconnue au sein d'un rectangle. Les auteurs relatent un taux moyen de réussite de $80 \%$ pour l'ensemble des utilisateurs.

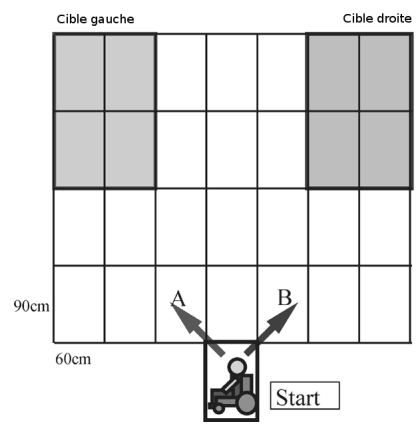

Figure 6. Déplacement du fauteuil piloté par l'interface BMI afin d'atteindre l'une ou l'autre des cibles (image extraite de Tanaka et al., 2005b).

Rebsamen et al., Université de Singapour ont développé récemment une interface destinée à piloter automatiquement les déplacements d'une personne lourdement handicapée au sein d'un immeuble d'un endroit à l'autre le long de chemins prédéfinis et balisés (Zeng et al., 2007). Pour aider des personnes atteintes du syndrome d'enfermement, les auteurs proposent d'adjoindre à leur système une interface BMI pour le choix des destinations (Rebsamen et al., 2007b), (Rebsamen et al., 2007a). L'interface BMI utilisée est de type non invasive synchrone basée sur la détection des ERPs cognitifs de type P300. Le fauteuil étant à l'arrêt dans une position donnée, les destinations que l'utilisateur peut atteindre à partir de cette position lui sont proposées sous forme d'une matrice $3 \times 3$ affichée à l'écran (voir figure 7 ). La phase de sélection d'une destination est réalisée à partir de 20 séquences d'intensifications de chacune des lignes et des colonnes de la matrice. Le fauteuil peut ensuite se diriger automatiquement vers la destination sélectionnée. Ce fauteuil, équipé de l'interface BMI et de l'interface de pilotage supervisé des déplacements, a été expérimenté par 5 personnes valides au sein du laboratoire de l'Université de Singapour, qui ont toutes réussi à atteindre la destination voulue dès leur premier essai.

del R. Millán et al., IDIAP, Martigny, Suisse proposent, dans le même ordre d'idée, de commander par la pensée le sens de déplacement d'un fauteuil roulant équipé d'une interface de conduite intégrant une stratégie collaborative adaptative, le niveau d'as- 


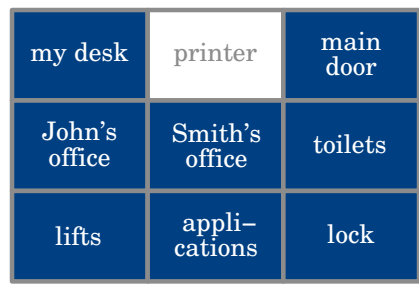

(a) pièces

\begin{tabular}{|c|c|c|}
\hline level 1 & level 2 & level 3 \\
\hline level 4 & level 5 & level 6 \\
\hline level 8 & $\begin{array}{c}\text { more } \\
\text { levels }\end{array}$ & $\begin{array}{c}\text { other } \\
\text { locations }\end{array}$ \\
\hline
\end{tabular}

(b) niveau

Figure 7. Interface utilisateur : matrices $3 \times 3$ de sélection de la destination (images extraites de Rebsamen et al.,2007b).

sistance du système étant évalué en temps-réel (Philips et al., 2007). L'intérêt d'une telle commande est illustré dans un environnement simulé dans lequel l'utilisateur tente de se déplacer à l'aide d'un fauteuil virtuel en empruntant un itinéraire consigné. L'interface BMI utilisée est de type non invasive asynchrone basée sur l'analyse des signaux EEG spontanés dans les gammes de fréquences 8-48 Hz. Durant la phase d'apprentissage, l'utilisateur effectue différentes tâches mentales parmi lesquelles le système sélectionne les trois plus discriminantes. A chacune de ces tâches sélectionnées est alors associée une commande : « tout droit », «à gauche » et «à droite ». Le système de reconnaissance des signaux EEG est chargé de reconnaître ces trois états mentaux en temps-réel et de fournir à l'interface de conduite du fauteuil la probabilité de sélection de chacune de ces trois commandes (Vanacker et al., 2007).

Ce système a été testé par deux volontaires valides. Les résultats des essais en environnement simulé montrent que les performances de l'interface BMI sont assez faibles puisque le taux de reconnaissance moyen pour les deux utilisateurs a été de 55 $\%$. Néanmoins, il est intéressant de noter qu'un taux de reconnaissance de $55 \%$ de l'interface BMI est suffisant pour piloter le fauteuil le long d'un couloir. Les auteurs ont également testé le fauteuil roulant équipé de l'interface de conduite et de l'interface BMI en environnement réel. Néanmoins, seuls les résultats en environnement simulé sont analysés dans (Galán et al., 2008).

Pfurtscheller et al., Université de Technologie de Graz, Autriche proposent une interface BMI permettant à l'utilisateur de naviguer en fauteuil roulant en environnement virtuel par simple imagination du mouvement du pied (Pfurtscheller et al., 2006b). Cette interface BMI est de type asynchrone non invasive basée sur la détection de l'activité oscillatoire sensorimotrice dans la bande $\beta$ lors de l'imagination du mouvement du pied, et ce à partir des signaux EEG extraits d'une seule électrode. L'utilisateur de cette interface est placé dans une rue virtuelle peuplée d'avatars. La tâche du patient consiste à se déplacer d'un avatar à l'autre jusqu'à la fin de la rue, en s'arrêtant au niveau de chaque avatar à l'intérieur d'une sphère de communication invisible permettant ainsi une communication orale avec ce dernier. Tant que l'utilisateur imagine le mouvement du pied, le fauteuil avance à vitesse constante, sinon il 


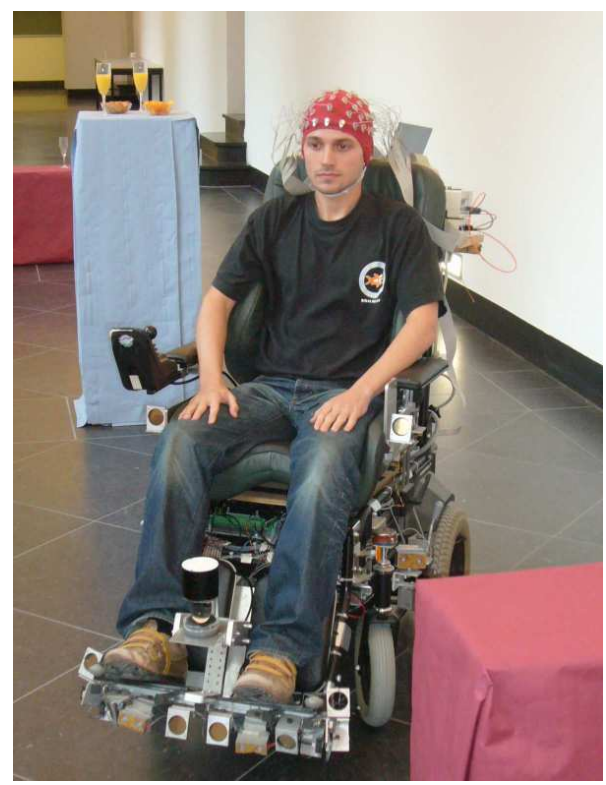

Figure 8. Fauteuil roulant développé par l'équipe IDIAP (image extraite de Vanacker et al., 2007).

s'arrête. Cette interface a été utilisée avec un succès de $90 \%$ par un patient tétraplégique (Leeb et al., 2007). Les $10 \%$ d'erreur sont dus à un arrêt soit trop près soit trop loin des avatars, le patient devant impérativement s'arrêter à l'intérieur des sphères successives. Face à ces résultats prometteurs, les auteurs cherchent à étendre leurs travaux en permettant à l'utilisateur de choisir aussi le sens de déplacement du fauteuil, et par la suite de transférer cette étude virtuelle sur un fauteuil réel.

\subsection{Contrôle d'une prothèse par une interface BMI}

Dans le cadre du développement d'interfaces BMI permettant le contrôle automatique de prothèses neuromotrices à partir de l'activité cérébrale, Chapin et al. ont été les premiers à suggérer la possibilité de contrôler en temps-réel des prothèses articulées à partir de la seule mesure des signaux électriques cérébraux par des techniques invasives (Chapin et al., 1999). Pour cela, les auteurs ont implanté des électrodes dans le cortex moteur de rats qui ont été entraînés à obtenir de l'eau à chaque fois qu'ils actionnaient une manette. Durant cette phase d'apprentissage, les auteurs ont enregistré les potentiels d'action de 46 neurones du cortex moteur des animaux, puis ils ont analysé et combiné ces signaux de manière à prédire la trajectoire $1 \mathrm{D}$ de la manette en fonction de l'activité neuronale des animaux (Wessberg et al., 2000). A l'issue de 
cette phase d'apprentissage, 4 des 6 animaux parvenaient à contrôler leurs signaux neuronaux de manière à obtenir de l'eau sans activer physiquement la manette.

Cette même stratégie d'apprentissage a été utilisée par Carmena et al., qui ont entraîné des singes à contrôler leur activité cérébrale motrice de manière à positionner virtuellement un curseur sur une cible ou à exercer virtuellement une force de préhension sur une manette (Carmena et al., 2003). Taylor et al. ont également entraîné deux singes à contrôler leur activité cérébrale de manière à réaliser des mouvements visant à atteindre une cible dans l'espace 3D (Taylor et al., 2003). Récemment, les chercheurs de cette même équipe ont montré l'intérêt de l'apprentissage basé sur l'observation et le contrôle de l'activité motrice en boucle fermée. Ils ont entraîné deux singes à contrôler leur activité cérébrale dans le but de piloter en temps-réel un bras robotisé à 5 degrés de liberté ( 3 au niveau de l'épaule, 1 au niveau du coude et un au niveau de la main) lors d'une tâche d'auto-alimentation (Velliste et al., 2008).

Ces recherches reposent sur une phase d'apprentissage au cours de laquelle des animaux sains, dont le cortex moteur est intact, ont réellement manipulé les actionneurs avant d'apprendre à les contrôler par leur seule activité cérébrale. Pour transposer les résultats cliniques obtenus sur des animaux sains au cas des personnes paralysées, il doit être prouvé que les signaux relatifs aux mouvements persistent dans le cortex moteur en dépit du handicap et que ces signaux sont générés par une intention de mouvement même lorsque des capteurs sensoriels et les mouvements des membres sont absents depuis longtemps (Donoghue et al., 2007).

Kennedy et al. ont montré que les signaux neuronaux, enregistrés à l'aide d'une électrode neurothrophique implantée dans le cortex moteur d'une patiente de 76 ans atteinte de SLA étaient restés stables et que cette patiente était capable de générer volontairement des potentiels d'actions dans son cortex moteur (Kennedy et al., 2000). Ces chercheurs ont montré qu'un deuxième patient, âgé de 51 ans et ayant été victime d'une attaque cérébrale 3 ans avant l'étude, était capable, au bout de 5 mois d'apprentissage, de contrôler ses potentiels corticaux lents (SCPS). Ce patient a appris à communiquer via une interface de communication palliative à la vitesse de 3 caractères par minute, puis à fléchir un doigt d'une main virtuelle (Kennedy et al., 2004). Ces expériences ont été realisées en capturant les potentiels d'action qui dépassent un certain seuil et en faisant correspondre à ces dépassements soit un déplacement de curseur soit une flexion en temps-réel d'un doigt artificiel.

Dans (Hochberg et al., 2006), les potentiels d'action et les SCPS sont observés à partir d'une matrice de 96 électrodes implantée dans le cortex moteur primaire de 2 patients agés de 25 et 55 ans atteints de tétraplégie. Cette étude a montré que 3 ans après l'accident moteur, l'activité cérébrale liée à l'intention de mouvements était toujours suffisante pour générer des potentiels d'action exploitables par l'interface BMI. De plus, l'observation de l'activité neuronale sur 96 électrodes a montré que les mouvements imaginés des membres modulaient les décharges neuronales sur de nombreuses électrodes. Cette expérimentation montre en outre que certains neurones sont sélectifs vis-à-vis du mouvement imaginé (main) alors que d'autres le sont moins et émettent des potentiels d'action lors de l'imagination d'actions très différentes. 
A l'issue de la phase d'apprentissage, l'un des patients a été capable de moduler son activité neuronale pour utiliser une BCI simulant l'ouverture d'emails, pour dessiner une figure circulaire en utilisant le logiciel « Paint », et pour piloter une interface domotique lui permettant d'ajuster le volume de la télévision, l'allumer, l'éteindre, ou changer de chaîne. Enfin, ce patient a pu contrôler un membre articulé lui permettant d'attraper un objet et de le déplacer d'un endroit à l'autre. Cet essai clinique pilote montre qu'une personne incapable de déplacer ou de sentir un membre paralysé peut malgré tout utiliser un prothèse neuromotrice commandée par sa seule activité neuronale pour réaliser certaines actions de la vie courante.

Bien que les signaux intracorticaux fournissent plus d'information sur la direction d'un mouvement de bras que ceux enregistrés à la surface du cortex, la mise en oeuvre d'une interface BMI de type invasive reste techniquement difficile et engendre des risques cliniques non négligeables. C'est pourquoi de nombreux auteurs se focalisent sur le développement de prothèses neuromotrices non invasives.

Guger et al. ont été les premiers auteurs à proposer une interface BMI couplée à une main artificielle, basée sur l'analyse des rythmes sensorimoteurs permettant la détection de l'imagination des mouvements des mains droite et gauche, testée uniquement par des individus valides (Guger et al., 1999). En 2000, Pfurtscheller et al. ont développé une interface BMI non invasive, basée sur l'analyse des rythmes sensorimoteurs, permettant de contrôler une orthèse électrique de la main (Pfurtscheller et $a l ., 2000$ a). Ce système a été utilisé par un patient tétraplégique de 22 ans qui a réussi, après 5 mois d'apprentissage, à fermer la prothèse en imaginant un mouvement du pied droit, et à ouvrir la prothèse en imaginant un mouvement de la main droite, et ce, avec une précision proche de $100 \%$. Les signaux EEG utilisés sont ceux enregistrés sur les électrodes $\mathrm{C} 3, \mathrm{C} 4$ et $\mathrm{Cz}$. Les signaux EEG échantillonnés à $128 \mathrm{~Hz}$ sont ensuite filtrés entre 0,5 et $30 \mathrm{~Hz}$. Le traitement des signaux filtrés est réalisé par un filtre adaptatif autorégressif et l'extraction des commandes en deux classes se fait par analyse discriminante linéaire.

En l'état, une telle prothèse ne permet pas d'attraper correctement un objet. En effet, la fonction de préhension nécessite de générer des mouvements des doigts et du pouce. Il est donc nécessaire d'accéder à 4 groupes de muscles que sont les extenseurs des doigts et du pouce pour ouvrir la main, les flexeurs de doigts pour fermer la main, le flexeur de pouce pour attraper l'objet, et l'extenseur du poignet pour stabiliser la main. C'est ainsi que Pfurtscheller et al. ont décomposé la prise et dépose d'un objet en 5 phases successives, et ont proposé une interface BMI couplée à un système de stimulation fonctionnelle électrique (FES), ce système étant capable de stimuler des muscles restés fonctionnels mais ne pouvant plus être commandés par le patient (Pfurtscheller et al., 2003). Au lieu d'utiliser des capteurs extérieurs localisés sur les muscles du patient, c'est l'interface BMI qui commande ici le passage d'une phase à l'autre du mouvement de préhension à chaque imagination d'un mouvement particulier par le patient.

Néanmoins, un tel système ne permet pas de stimuler des muscles profonds, et il est très difficile d'obtenir une stabilité de la main indépendante de la position du 
préhenseur. C'est pourquoi le patient a été équipé d'une orthèse mécanique fixant le poignet dans une position dorsale fléchie, laquelle assure la stabilité nécessaire à la préhension d'un objet (Pfurtscheller et al., 2005). Ainsi équipé le patient a pu, pour la première fois depuis son accident, boire un verre d'eau sans aucune aide et sans utiliser une paille. Les auteurs ont également montré qu'une courte phase d'apprentissage de 3 jours avait été suffisante pour qu'un autre patient tétraplégique de 42 ans parvienne à piloter une neuroprothèse implantée (système Freehand) via cette interface BMI non invasive (Müller et al., 2005). Le signal de sortie de l'interface BMI a été utilisé pour indiquer au stimulateur Freehand les changements d'étape dans la réalisation du mouvement de préhension, celui-ci étant décomposé en 3 étapes successives.

Cette expérience confirme que la rééducation motrice pourrait être un nouveau domaine d'application des BMI en milieu clinique. Les électrodes implantées sur la duremère nous ont permis de récupérer des signaux de très bonne qualité, il est donc fort probable que la discrimination des mouvements soit facilitée avec ce type signaux. Les BMI faiblement invasives pourraient alors constituer un compromis intéressant dans l'attente soit de BMI non invasives plus performantes hors laboratoire de recherches, soit de BMI invasives plus robustes et mieux tolérees par le patient.

\subsection{Interface cerveau-ordinateur et approche thérapeutique}

Un domaine récent d'application des BMI est l'étude des mécanismes de fonctionnement normal, de dysfonctionnement et de récupération ainsi que l'aide au diagnostic et à la rééducation fonctionnelle (Stokes et al., 2006). Dans le cas d'une BMI de type synchrone, les potentiels évoqués permettent d'étudier la récupération des mécanismes neuronaux liés au langage chez des patients aphasiques suite à un accident vasculaire cérébral (Pulvermuller et al., 2004). Avec une BMI de type asynchrone, le contrôle des potentiels corticaux lents permet, dans certains cas, de réduire les crises d'épilepsie (Kotchoubey et al., 2001). Les interfaces cerveau-machine peuvent aussi avoir une valeur en rééducation neuronale, pour les patients sévérement handicapés, en renforçant l'usage des chemins neuronaux endommagés en tirant parti de la plasticité du système nerveux (Dobkin, 2007). Enfin l'utilisation des interfaces cerveau-machine pour la rééducation motrice ne doit pas être négligée (Birbaumer $e t$ al., 2007), (Soekadar et al., 2008).

Très récemment, une interface cerveau-machine a été utilisée comme outil d'aide à la récupération motrice (mouvement de la main) d'un patient atteint d'une hémiparésie du côté droit (tout en gardant un contrôle partiel du membre supérieur droit, et notamment de la main) et souffrant de douleurs neuropathiques résistantes aux traitements médicamenteux (Van Langhenhove et al., 2008b). Cela consiste à placer des électrodes sur la dure-mère, au dessus des zones sensori-motrices du cortex qui correspondent aux membres douloureux, afin de les stimuler électriquement. Cette stimulation a pour conséquence la diminution totale ou partielle des douleurs. 
Nous avons temporairement utilisé les électrodes de stimulation implantées ici de manière faiblement invasive, pour mesurer l'activité cérébrale du patient et l'utiliser pour piloter une interface cerveau-ordinateur portable (Van Langhenhove et al., 2007). Dans ce contexte précis, la BMI a été utilisée comme un outil de rééducation permettant au patient d'apprendre à contrôler l'activité motrice de sa main pour qu'il en retrouve l'usage.

Plus précisément, l'interface BMI isole le rythme $\mu$ dans le signal correspondant à l'aire motrice de la main déficiente, et fournit au patient, par un feedback visuel, une représentation imagée de la force du mouvement de la main qu'il effectue. Le déplacement vertical d'un simple curseur suffit à cette représentation. Quand la main handicapée est immobile, le curseur est en bas de l'écran. Dès que le patient commence à fermer la main, le curseur monte. Si le patient maintient le mouvement (main fermée), le curseur atteint le haut de l'écran. Plus le mouvement est performant, en termes de force et de rapidité, plus la puissance du rythme $\mu$ diminue, ce qui accélère la montée du curseur vers le haut de l'écran. Durant une séance, le sujet réalise plusieurs tentatives de mouvement, avec ou sans le feedback de la BMI. Lors des tentatives avec feedback, la présence de bouffées distinctes de forte puissance dans les hautes fréquences du spectre est constatée (liées à l'intensité de la force de fermeture de la main). Ces bouffées n'apparaissent pas ou elles sont nettement moins distinctes lors des tentatives effectuées sans le feedback de la BMI. Parallèlement, l'observation visuelle du patient a montré qu'en présence du feedback les mouvements semblent plus complets et rapides alors qu'ils semblent plus laborieux et moins puissants sans ce feedback. Cette expérience semble montrer que la rééducation motrice pourrait être un nouveau domaine d'application des BMI en milieu clinique (Van Langhenhove et al., 2008a).

\section{Etat actuel des recherches}

A l'heure actuelle, les BMIs constituent un domaine de recherche en pleine expansion. Dans le monde, environ six laboratoires étaient identifiés comme travaillant dans ce domaine en 1994. En 1999, lors du premier congrès international sur les BMI, les chercheurs étaient issus de 38 laboratoires différents. Au workshop organisé en 2005 par le Wadsworth Center, plus de 100 laboratoires étaient représentés.

On peut identifier quelques équipes pionnières : le laboratoire des désordres du système nerveux du Wadsworth Center (Albany, NY, USA, équipe de Jonathan Wolpaw $^{1}$ ); l'institut de psychologie médicale et de neurobiologie comportementale de l'Université de Tüebingen (Allemagne, équipe de Niels Birbaumer ${ }^{2}$ ); le département d'informatique médicale de l'Université de technologie de Graz (Autriche, équipe de

1. http ://www.bciresearch.org/

2. http ://www.mp.uni-tuebingen.de/ 
Gert Pfurtscheller ${ }^{3}$ ) ; le groupe Intelligent Data Analysis de Fraunhofer FIRST (Berlin, Allemagne, équipe de Klaus-Robert Müller $\left.{ }^{4}\right)$.

Ces équipes ont collaboré durant de nombreuses années, soit par l'intermédiaire d'échanges de chercheurs, soit dans le cadre de programmes de recherche internationaux. Un résultat tangible de cette collaboration est le logiciel BCI2000 (Schalk et al., 2004), plate-forme expérimentale d'enregistrement et de traitement des signaux EEG, qui est disponible gratuitement pour les autres groupes de recherche ${ }^{5}$.

Au niveau européen, plusieurs projets relatifs aux BMIs ont été financés, principalement dans le cadre du programme Future and Emerging Technologies 6 . On peut citer par exemple le projet PRESENCE, qui a visé à développer des systèmes avancés de réalité virtuelle, le projet OPTIVIP qui a étudié le champ des prothèses invasives, ou encore le projet Neurobotics 7 qui intègre un volet sur le développement de neuroprothèses de type BMI. Plus récemment, le projet MAIA ${ }^{8}$ a permis de fédérer les travaux de cinq laboratoires européens en ciblant directement le domaine des interfaces cerveau-machine. Certains réseaux d'excellence, principalement PASCAL et SIMILAR, sont également très impliqués dans la recherche sur les BMIs. Les projets d'étendue européenne sont bien adaptés au développement de collaborations entre des laboratoires multidisciplinaires, dont le domaine de compétence s'étend des neurosciences et du traitement de signal jusqu'à la communication palliative.

En France, les activités de recherche liées aux BMIs commencent à se mener de façon concertée, du fait que de nombreux laboratoires ont développé de solides compétences durant la dernière décennie. La première réunion de portée nationale sur les BMIs, qui a eu lieu à Lille en avril 2006, a regroupé plus de quarante chercheurs issus de quinze laboratoires 9 . Ces équipes de recherche structurent actuellement leurs activités, par l'intermédiaire de réunions académiques — notamment grâce au soutien du GDR STIC-Santét ${ }^{\sqrt{10}}$ et de l'IFRATH ${ }^{11}$ — ou grâce à des projets de recherche financés par l'ANR, tels que le projet OpenVIBE ${ }^{12}$.

\section{Perspectives}

Bien que les recherches sur les aspects fondamentaux aient rapidement progressé durant les dernières années, très peu d'expérimentations ont été menées en dehors du cadre clinique. Grâce au soutien de la fondation Altran ${ }^{13}$, les chercheurs du Wad-

\footnotetext{
3. http ://bci.tugraz.at/

4. http ://ida.first.fraunhofer.de/homepages/ida/

5. http ://www.bci2000.org/

6 . http ://cordis.europa.eu/ist/fet/home.html

7. http ://www.neurobotics.org/

8. http ://www.maia-project.org/

9. http ://www-bci.univ-lille1.fr/

10. http ://stic-sante.org/

11. http ://ifrath.free.fr/

12. http ://www.irisa.fr/siames/OpenViBE/

13. http ://www.fondation-altran.org/
} 
sworth Center ont développé une interface BMI qui est testée depuis juin 2006 sur plusieurs patients. A notre connaissance, ce genre d'expérimentation n'a pas encore été réalisé en France. Comment faire pour que cette nouvelle technique de palliation du handicap sorte des laboratoires?

Il faut se poser la question de l'intérêt que présentent les BMI pour les chercheurs des différentes disciplines. Dans la communauté STIC (Sciences et Technologies de l'Information et de la Communication), de nombreux chercheurs sont principalement attirés par la complexité des données électroencéphalographiques recueillies lors des expérimentations BMI. Ces données multidimensionnelles, très bruitées et non stationnaires, sont parfaites pour tester et valider des techniques avancées de segmentation et de classification. A l'opposé, pour de nombreux chercheurs de la communauté médicale, une interface BMI est une technique supplémentaire d'imagerie fonctionnelle à la fois non invasive, peu coûteuse et d'emploi facile. De ce fait, une expérimentation BMI leur permet de confirmer ou d'infirmer rapidement des hypothèses grâce à l'observation de corrélations entre une tâche réalisée par l'utilisateur et les variations de son activité cérébrale.

Si les différentes communautés collaborant au développement des BMI se fixaient des objectifs communs, il ne fait nul doute que la recherche sur les BMI changerait sensiblement de cap. Pour aller dans ce sens, une solution consiste à placer systématiquement le patient handicapé au coeur du problème, en vue de définir quelles techniques BMI peuvent dès maintenant être mises à sa disposition, que ce soit dans un cadre clinique ou à domicile.

\section{Remerciements}

Les auteurs remercient la Région Nord-Pas de Calais et l'IRRH (Institut Régional de Recherche sur le Handicap) pour l'aide financière accordée à ce projet, ainsi que les collègues du Wadsworth Center pour le constant soutien qu'il nous ont apporté depuis le début de notre collaboration.

\section{Bibliographie}

Bashashati A., Fatourechi M., Ward R. K., Birch G. E., « A survey of signal processing algorithms in brain-computer interfaces based on electrical brain signals », Journal of Neural Engineering, vol. 4, $\mathrm{n}^{\circ}$ 2, p. R32-R57, 2007.

Bayliss J. D., « Use of the Evoked Potential P3 Component for control in a virtual apartment », IEEE Transactions on Neural Systems and Rehabilitation Engineering, vol. 11, n 2, p. 113 116, June, 2003.

Besserve M., Martinerie J., Garnero L., « Non-invasive classification of cortical activities for brain computer interface : A variable selection approach », 5th IEEE International Symposium on Biomedical Imaging : From Nano to Macro, ISBI 2008, Paris, p. 1063-1066, May, 2008. 
Birbaumer N., Cohen L. G., « Brain-computer interfaces : communication and restoration of movement in paralysis », The Journal of Physiology, vol. 579, n 3, p. 621-636, 2007.

Birbaumer N., Kubler A., Ghanayim N., Hinterberger T., Perelmouter J., Kaiser J., Iversen I., Kotchoubey B., Neumann N., Flor H., « The thought translation device (TTD) for completely paralyzed patients », IEEE Transactions on Rehabilitation Engineering, vol. 8, $\mathrm{n}^{\circ} 2$, p. 190-193, 2000.

Blankertz B., Dornhege G., Krauledat M., Müller K. R., Kunzmann V., Losch F., Curio G., « The Berlin Brain-Computer Interface : EEG-based communication without subject training », IEEE Transactions on Neural Systems and Rehabilitation Engineering, vol. 14, $\mathrm{n}^{\circ} 2$, p. 147-152, 2006 .

Blankertz B., Dornhege G., Krauledat M., Schröder M., Williamson J., Murray-Smith R., Müller K. R., « The Berlin Brain-Computer Interface presents the novel mental typewriter Hexo-Spell », Proceedings of the third International Workshop and Training course on BrainComputer Interfaces, Graz, Austria, p. 108-109, 2006b.

Blankertz B., Krauledat M., Dornhege G., Williamson J., Murray-Smith R., Müller K. R., « A note on brain actuated spelling with the Berlin Brain-Computer Interface », in C. Stephanidis (ed.), Proceedings of the 4th International Conference on Universal Access in HumanComputer Interaction (UAHCI'07) Held as Part of HCI International 2007 , Part II, vol. 4555, Springer, Beijing, China, p. 759-768, July, 2007.

Carmena J., Lebedev M., Crist R. E., O’Doherty J. E., Santucci D. M., Dimitrov D. F., Patil P. G., Henriquez C. S., Nicolelis M. A. L., « Learning to Control a Brain-Machine Interface for Reaching and Grasping by Primates », PLoS Biology, vol. 1, p. 193-208, 2003.

Chapin J. K., Moxon K. A., Markowitz R. S., Nicolelis M. A. L., « Real-time control of a robot arm using simultaneously recorded neurons in the motor cortex », Nature Neuroscience, vol. 2, p. 664-670, 1999

Congedo M., Lotte F., Lécuyer A., « Classification of movement intention by spatially filtered electromagnetic inverse solutions », Physics in Medicine and Biology, vol. 51, p. 1971-1989, 2006.

del R. Millán J., Mouriño J., Franzé M., Cincotti F., Varsta M., Heikkonen J., Babiloni F., « A Local Neural Classifier for the Recognition of EEG Patterns Associated to Mental Tasks », IEEE Transactions on Neural Networks, vol. 13, $\mathrm{n}^{\circ}$ 3, p. 678-686, March, 2002.

Dobkin B. H., « Brain-computer interface technology as a tool to augment plasticity and outcomes for neurological rehabilitation », The Journal of Physiology, vol. 579, $\mathrm{n}^{\circ}$ 3, p. 637642, 2007.

Donchin E., Spencer K. M., Wijesinghe R., « The mental prosthesis : assessing the speed of a P300-based computer interface », IEEE Transactions on Rehabilitation Engineering, vol. 8, $\mathrm{n}^{\circ} 2$, p. 174-179, 2000 .

Donoghue J., Nurmikko A., M M. B., Hochberg L., « Assistive technology and robotic control using MI ensemble-based neural interface systems in humans with tetraplegia », Journal of Physiology, vol. 579, p. 603-611, 2007.

Farwell L. A., Donchin E., « Talking Off The Top Of Your Head : A Mental Prosthesis Utilizing Event-Related Brain Potentials », Electroencephalography and Clinical Neurophysiology, vol. 70, p. 510-513, 1988.

Felton E. A., Lewis N. L., Wills S. A., Radwin R. G., Williams J. C., « Neural Signal Based Control of the DASHER Writing System », Proceedings of the 3rd International 
IEEE/EMBS Conference on Neural Engineering, The Fairmont Orchid, Kohala Coast, Hawaï, p. 366-370, May, 2007.

Friman O., Lüth T., Volosyak I., Gräser A., « Spelling with Steady-State Visual Evoked Potentials », Proceedings of the 3rd International IEEE/EMBS Conference on Neural Engineering, The Fairmont Orchid, Kohala Coast, Hawaï, p. 354-357, May, 2007.

Galán F., Nuttin M., Lew E., Ferrez P. W., Vanacker G., Philips J., del R. Millán J., « A Brainactuated wheelchair : Asynchronous and non-invasive Brain-computer interfaces for continuous control of robots », Clinical Neurophysiology, vol. 119, p. 2159-2169, 2008.

Gao X., Xu D., Cheng M., Gao S., « A BCI-Based Environmental Controller for the MotionDisabled », IEEE Transactions on Neural Systems and Rehabilitation Engineering, vol. 11, $\mathrm{n}^{\circ} 2$, p. 137-140, June, 2003.

Georgopoulos A. P., Langheim F. J. P., Leuthold A. C., Merkle A. N., « Magnetoencephalographic signals predict movement trajectory in space », Experimental Brain Research, vol. $167, \mathrm{n}^{\circ} 1$, p. 132-135, 2005.

Guger C., Harkam W., Hertnaes C., Pfurtscheller G., « Prosthetic control by an EEG-based brain-computer interface (BCI) », Proceedings of the 5th European Conference for the Advancement of Assistive Technology, Düsseldorf, Germany, November, 1999.

Guger C., Schlög A., Neuper C., Walterspacher D., Strein T., Pfurtscheller G., « Rapid prototyping of an EEG-based brain-computer interface (BCI) », IEEE Transactions on Rehabilitation Engineering, vol. 9, p. 49-58, 2001.

Hinterberger T., Mellinger J., Birbaumer N., « The Thought Translation Device : Structure of a multimodal brain-computer communication system », Proceedings of the 1st International IEEE/EMBS Conference on Neural Engineering, Capri Island, Italy, p. 603-606, March, 2003.

Hochberg L., Serruya M., Friehs G., Mukand J., Saleh M., Caplan A., Branner A., Chen D., Penn R., Donoghue J., « Neuronal ensemble control of prosthetic devices by a human with tetraplegia », Nature, vol. 442, p. 164-171, 2006.

Jerbi K., O.Bertrand, Schoendorff B., Hoffmann D., Minotti L., Kahane P., Berthoz A., Lachaux J.-P., « Online Detection of Gamma Oscillations in Ongoing Intracerebral Recordings : From Functional Mapping to Brain Computer Interfaces », Proceedings of the 6th International Symposium on Noninvasive Functional Source Imaging of the Brain and Heart and the International Conference on Functional Biomedical Imaging, NFSI-ISBI, Hangzhou,China, p. 330-333, october, 2007.

Kennedy P., Bakay R., Moore M., Adams K., Goldwaithe J., « Direct control of a computer from the human central nervous system », IEEE Transaction on Rehabilitation Engineering, vol. 8, p. 198-202, 2000.

Kennedy P. R., Kirby M. T., Moore M., King B., Mallory A., « Computer control using human Intracortical Local Field Potentials », IEEE Transaction on Neural Systems and Rehabilitation Engineering, vol. 12, p. 339-344, 2004.

Kotchoubey B., Strehl U., Uhlmann C., Holzapfel S., König M., Fröscher W., Blankenhorn V., Birbaumer N., " Modification of slow cortical potentials in patients with refractory epilepsy : a controlled outcome study », Epilepsia, vol. 42, n 3, p. 406-416, 2001.

Krusienski D. J., Sellers E. W., Cabestaing F., Bayoudh S., McFarland D. J., Vaughan T. M., Wolpaw J. R., « A comparison of classification techniques for the P300 Speller », Journal of Neural Engineering, vol. 3, n 4, p. 299-305, December, 2006. 
Le Pavec J., Clerc M., « Adaptive classification of mental states for asynchronous brain computer interfaces », Deuxième conférence française de Neurosciences Computationnelles, "Neurocomp08", Marseille France, October, 2008.

Lebedev M. A., Nicolelis M. A., « Brain-machine interfaces : past, present and future », TRENDS in Neurosciences, vol. 29, $\mathrm{n}^{\circ}$ 9, p. 536-546, 2006.

Leeb R., Friedman D., Müller-Putz G. R., Scherer R., Slater M., Pfurtscheller G., « Self-Paced (Asynchronous) BCI Control of a Wheelchair in Virtual Environments : A Case Study with a Tetraplegic », Computational Intelligence and Neuroscience Archive, vol. 2007, $\mathrm{n}^{\circ}$ 2, p. 18, 2007.

Li H., Li Y., Guan C., « An Effective BCI Speller Based on Semi-supervised Learning », Proceedings of the 28th Annual International Conference of the IEEE Engineering in Medicine and Biology Society, Marriott Marquis, New York, USA, p. 1161-1164, September, 2006.

Liang N., Bougrain L., « Averaging techniques for single-trial analysis of oddball event-related potentials », 4th International Brain-Computer Interface workshop, Graz Autriche, 2008.

Lotte F., Congedo M., Lécuyer A., Lamarche F., Arnaldi B., « A review of classification algorithms for EEG-based brain-computer interfaces », Journal of Neural Engineering, vol. 4, $\mathrm{n}^{\circ}$ 2, p. R1-R13, 2007.

Lüth T., Ojdanic D., Friman O., Prenzel O., Gräser A., « Low level control in a semiautonomous rehabilitation robotic system via a Brain-Computer Interface », Proceedings of the IEEE 10th International Conference on Rehabilitation Robotics (ICORR'07), Noordwijk, The Netherlands, p. 721-728, June, 2007.

Mason S. G., Bashashati A., Fatourechi M., Navarro K., Birch G., « A Comprehensive Survey of Brain Interface Technology Designs », Annals of Biomedical Engineering, vol. 35, $\mathrm{n}^{\circ} 2$, p. 137-169, February, 2007.

McFarland D. J., McCane L. M., David S. V., Wolpaw J. R., « Spatial filter selection for EEGbased communication », Electroencephalography and clinical neurophysiology, vol. 103, n 3, p. 386-394, 1997.

McFarland D. J., Miner L. A., Vaughan T. M., Wolpaw J. R., « Mu and Beta Rhythm Topographies During Motor Imagery and Actual Movements », Brain Topography, vol. 12, p. 177-186, 2000.

Mellinger J., Schalk G., Braun C., Preissl H., Rosenstiel W., Birbaumer N., Kübler A., « An MEG-based brain-computer interface (BCI)», NeuroImage, vol. 36, n 3, p. 581 - 593, 2007.

Müller G., Scherer R., Pfurtscheller G., Rupp R., « EEG-based neuroprosthesis control : a step towards clinical practice », Neuroscience Letters, vol. 382, n 1-2, p. 169-174, 2005.

Müller-Putz G. R., Pfurtscheller G., « Control of an Electrical Prosthesis with an SSVEP-Based BCI », IEEE Transactions on Biomedical Engineering, vol. 55, $\mathrm{n}^{\circ}$ 1, p. 361-364, January, 2008.

Obermaier B., Müller G. R., Pfurtscheller G., « 'Virtual Keyboard' Controlled by Spontaneous EEG Activity », IEEE Transactions on Neural Systems and Rehabilitation Engineering, vol. $11, n^{\circ} 4$, p. 422-426, December, 2003.

Pascual-Marqui R. D., « Standardized low-resolution brain electromagnetic tomography (sLORETA) : technical details », Methods and findings in experimental and clinical pharmacology, vol. 24, n Suppl-D, p. 5-12, 2002. 
Pfurtscheller G., Graimann B., Neuper C., « EEG-Based Brain-Computer Interface System », Wiley Encyclopedia of Biomedical Engineering, 2006a.

Pfurtscheller G., Guger C., Müller G., Krausz G., Neuper C., « Brain oscillations control hand orthosis in a tetraplegic », Neuroscience Letters, vol. 292, n 3, p. 211-214, 2000a.

Pfurtscheller G., Leeb R., Keinrath C., Friedman D., Neuper C., Guger C., Slater M., « Walking from thought », Brain Research, vol. 1071, $\mathrm{n}^{\circ}$ 1, p. 145-152, February, $2006 \mathrm{~b}$.

Pfurtscheller G., Müller G., Pfurtscheller J., Gerner H., Rupp R., « 'Thought' - control of functional electrical stimulation to restore hand grasp in a patient with tetraplegia », $\mathrm{Neu}$ roscience Letters, vol. 351, $\mathrm{n}^{\circ}$ 1, p. 33-36, 2003.

Pfurtscheller G., Müller-Putz G. R., Pfurtscheller J., Rupp R., « EEG-Based Asynchronous BCI Control Functional Electrical Stimulation in a Tetraplegic Patient », EURASIP Journal on Applied Signal Processing, vol. 19, p. 3152-3155, 2005.

Pfurtscheller G., Neuper C., Guger C., Harkam W., Ramoser H., Schlögl A., Obermaier B., Pregenzer M., « Current trends in Graz brain-computer-interface (BCI) research », IEEE Transactions on Rehabilitation Engineering, vol. 8, p. 216-219, $2000 \mathrm{~b}$.

Pham M., Hinterberger T., Neumann N., Kübler A., Hofmayer N., Grether A., Wilhelm B., Vatine J. J., Birbaumer N., « An Auditory Brain-Computer Interface Based on the SelfRegulation of Slow Cortical Potentials », Neurorehabilitation and Neural Repair, vol. 19, $\mathrm{n}^{\circ}$ 3, p. 206-218, 2005.

Philips J., del R. Millán J., Vanacker G., Lew E., Galán Moles F., Ferrez P. W., Van Brussel H., Nuttin M., « Adaptive Shared Control of a Brain-Actuated Simulated Wheelchair », Proceedings of the IEEE 10th International Conference on Rehabilitation Robotics (ICORR'07), Noordwijk, The Netherlands, p. 408-414, june, 2007.

Pulvermuller F., Mohr B., Lutzenbergers W., « Neurophysiological correlates of word and pseudo-word processing in well-recovered aphasics and patients with right-hemispheric stroke », Psychophysiology, vol. 41, n 4, p. 584-591, 2004.

Rakotomamonjy A., Guigue V., Mallet G., Alvarado V., « Ensemble of SVMs for Improving Brain Computer Interface P300 Speller Performances », Proceedings of ICANN'2005, Lecture Notes in Computer Science, vol. 3696, p. 45-50, 2005.

Rebsamen B., Burdet E., Guan C., Leong Teo C., Zeng Q., Ang M., Laugier C., " Controlling a Wheelchair using a BCI with low information transfer rate », Proceedings of the IEEE 10th International Conference on Rehabilitation Robotics (ICORR'07), Noordwijk, The Netherlands, p. 1003-1008, June, 2007a.

Rebsamen B., Teo C. L., Zeng Q., Ang Jr. M. H., Burdet E., Guan C., Zhang H., Laugier C., « Controlling a Wheelchair Indoors Using Thought», IEEE Computer Society, vol. 70, p. 510-513, 2007b.

Schalk G., McFarland D. J., Hinterberger T., Birbaumer N., Wolpaw J. R., « BCI2000 : A general-purpose brain-computer interface (BCI) system », IEEE Transactions on Biomedical Engineering, vol. 51, p. 1034-1043, 2004.

Scherer R., Müller G. R., Neuper C., Graimann B., Pfurtscheller G., « An Asynchronously Controlled EEG-Based Virtual Keyboard : Improvement of the spelling rate », IEEE Transactions on Biomedical Engineering, vol. 51, n 6, p. 979-984, 2004.

Schwartz A., Cui X. T., Weber D., Moran D. W., « Brain Controlled Interfaces : Movement restoration with Neural Prosthetics », Neuron, vol. 52, p. 205-220, 2006. 
Sellers E., Krusienski D. J., McFarland D. J., Vaughan T. M., Wolpaw J. R., « A P300 eventrelated potential brain-computer interface $(\mathrm{BCI})$ : The effects of matrix size and inter stimulus interval on performance », Biological Psychology, vol. 73, n 3, p. 242-252, 2006a.

Sellers E. W., Donchin E., « A P300-based brain computer interface : Initial tests by ALS patients », Clinical Neurophysiology, vol. 117, p. 538-548, 2006b.

Sitaram R., Weiskopf N., Caria A., Veit R., Erb M., Birbaumer N., « fMRI Brain-Computer Interfaces », Signal Processing Magazine, IEEE, vol. 25, n 1, p. 95-106, 2008.

Soekadar S., Haagen K., Birbaumer N., Brain Computer Interfaces (BCI) : Restoration of movement and Thought from Neuroelectric and Metabolic Brain Activity, Coordination : Neural, Behavioral and Social Dynamics, Springer Berlin Heidelberg, chapter 11, p. 229-252, 2008.

Souères P., Jouffrais C., Celebrini S., Trotter Y., « Robotics Insights for the Modeling of Visually Guided Hand Movements in Primates », Lecture Notes in Control and Information Sciences, Biology and Control Theory : Current Challenges, vol. 357/2007, p. 53-75, 2007.

Stokes M., James C. J., « W9 Brain-Computer Interfacing (BCI) in rehabilitation », Workshops/Clinical Neurophysiology, vol. 117, p. S25-S31, 2006.

Tanaka K., Matsunaga K., Hori S., « Electroencephalogram-Based Control of a Mobile Robot», Electrical Engeeniring in Japan - Wiley InterScience, vol. 152, n 3, p. 39-46, August, 2005a.

Tanaka K., Matsunaga K., Wang H. O., « Electroencephalogram-Based Control of an Electric Wheelchair », IEEE Transactions on Robotics, vol. 21, n 4, p. 762-766, $2005 \mathrm{~b}$.

Taylor D. M., Tillery S. I. H., Schwartz A. B., « Information conveyed through brain-control : cursor versus robot », IEEE Transactions on Neural Systems and Rehabilitation Engineering, vol. 11, $\mathrm{n}^{\circ} 2$, p. 195-199, 2003.

Van Langhenhove A., Bekaert M.-H., Cabestaing F., « Vers une BCI utilisable en dehors du milieu clinique », in CEPADUES-EDITIONS (ed.), Conférence Internationale Sur l'Accessibilité et les systèmes de suppléance aux personnes en situation de handicaps, ASSISTH'2007, Toulouse, p. 369-375, November, 2007.

Van Langhenhove A., Bekaert M.-H., Cabestaing F., Nguyen J.-P., « Interfaces cerveauordinateur et rééducation fonctionnelle : étude de cas chez un patient hémiparésique », Sciences et Technologies pour le Handicap, vol. 2/1, p. 41-54, 2008 a.

Van Langhenhove A., Bekaert M.-H., Nguyen J.-P., « Utilisations thérapeutiques des interfaces cerveau-ordinateur», HANDICAP 2008, Hôtel Mercure, Porte de Versailles Vaugirard, Paris (FRANCE), June, 2008b.

Vanacker G., del R. Millán J., Lew E., Ferrez P. W., Galán Moles F., Philips J., Van Brussel H., Nuttin M., « Context-Based Filtering for Assisted Brain-Actuated Wheelchair Driving », Computational Intelligence and Neuroscience Archive, 2007.

Velliste M., Perel S., Spalding M. C., Whitford A. S., Schwartz A. B., « Cortical control of a prosthetic arm for self-feeding », Nature, vol. 453, p. 1098-1101, 2008.

Wang C., Guan C., Zhang H., « P300 Brain-Computer Interface Design for Communication and Control Applications », Proceedings of the 2005 IEEE Engineering in Medicine and Biology 27th Annual Conference, Shanghai - China, September, 2005.

Ward D. J., Blackwell A. F., MacKay D. J. C., « DASHER : a data entry interface using continuous gestures and language models », Proceedings of the 13th Annual ACM Symposium on User Interface Software and Technology, San Diego, California, USA, p. 129 - 137, November, 2000. 
Wei W., Gao X., Gao S., « One-Versus-the-Rest(OVR) Algorithm : An Extension of Common Spatial Patterns(CSP) Algorithm to Multi-class Case », Proceedings of 27th Annual International Conference of the Engineering in Medicine and Biology Society, IEEE-EMBS 2005, p. 2387-2390, 2005.

Wessberg J., Stambaugh C. R., Kralik J. D., Beck P. D., Laubach M., Chapin J. K., Kim J., Biggs S. J., Srinivasan M. A., Nicolelis M. A. L., « Real-time prediction of hand trajectory by ensembles of cortical neurons in primates », Nature, vol. 408, p. 361-365, 2000.

Wills S. A., MacKay D. J. C., « DASHER : An Efficient Writing System for Brain-Computer Interfaces? », IEEE Transactions on Neural Systems and Rehabilitation Engineering, vol. 14, n 2, p. 244-246, 2006.

Wolpaw J. R., Birbaumer N., McFarland D. J., Pfurtscheller G., Vaughan T. M., « Braincomputer interfaces for communication and control », Clinical Neurophysiology, vol. 113, p. 767-791, 2002.

Wolpaw J. R., McFarland D. J., « Control of a two-dimensional movement signal by a noninvasive brain-computer interface in humans », Proceedings of the National Academy of Science of the USA, vol. 101, $\mathrm{n}^{\circ}$ 51, p. 17849-17854, December, 2004.

Wolpaw J. R., McFarland D. J., Vaughan T. M., Schalk G., « The Wadsworth Center braincomputer interface (BCI) research and development program », IEEE Transactions on Neural Systems and Rehabilitation Engineering, vol. 11, p. 1-4, june, 2000.

Zeng Q., Burdet E., Rebsamen B., Teo C. L., « Evaluation of the Collaborative Wheelchair Assistant System », Proceedings of the IEEE 10th International Conference on Rehabilitation Robotics (ICORR'07), Noordwijk, The Netherlands, p. 601-608, June, 2007. 